\title{
Study of turbulent heat transfer of aviation kerosene flows in a curved pipe at supercritical pressure
}

\author{
Xunfeng Li ${ }^{\text {a }}$, Fengquan Zhong ${ }^{b}$, Xuejun Fan $^{\text {b }}$, Xiulan Huai ${ }^{\mathrm{a}, *}$, Jun Cai ${ }^{\mathrm{a}}$ \\ a Institute of Engineering Thermophysics, Chinese Academy of Sciences, P.O. Box 2706, Beijing 100190, China \\ ${ }^{\mathrm{b}}$ Institute of Mechanics, Chinese Academy of Sciences, Beijing 100190, China
}

\section{A R T I C L E I N F O}

Article history:

Received 30 November 2009

Accepted 20 April 2010

Available online 28 April 2010

\section{Keywords:}

Aviation kerosene

Supercritical pressure

Body force

Convective heat transfer

Numerical study

\begin{abstract}
A B S T R A C T
The flow and heat transfer characteristics of China No. 3 aviation kerosene in a heated curved tube under supercritical pressure are numerically investigated by a finite volume method. A two-layer turbulence model, consisting of the RNG $k-\epsilon$ two-equation model and the Wolfstein one-equation model, is used for the simulation of turbulence. A 10-species kerosene surrogate model and the NIST Supertrapp software are applied to obtain the thermophysical and transport properties of the kerosene at various temperature under a supercritical pressure of $4 \mathrm{MPa}$. The large variation of thermophysical properties of the kerosene at the supercritical pressure make the flow and heat transfer more complicated, especially under the effects of buoyancy and centrifugal force. The centrifugal force enhances the heat transfer, but also increases the friction factors. The rise of the velocity caused by the variation of the density does not enhance the effects of the centrifugal force when the curvature ratios are less than 0.05 . On the contrary, the variation of the density increases the effects of the buoyancy.
\end{abstract}

( 2010 Elsevier Ltd. All rights reserved.

\section{Introduction}

Supercritical fluid has been applied broadly in aeronautics, astronautics and nuclear reactor due to its excellent heat transfer properties $[1,2]$. The specific heat capacity of supercritical fluid has a peak value when it varies with temperature at a supercritical pressure. The temperature corresponding to the peak point is called pseudo-critical temperature at that supercritical pressure. The physical properties of supercritical fluid, such as specific heat capacity and thermal conductivity, have a large variation near the pseudo-critical point corresponding to a supercritical pressure, which induces non-conventional heat transfer. While the significant change of the density near the pseudo-critical point can cause a strong secondary flow under the effects of body force, which enhances the convective heat transfer. There were many experimental and numerical studies on the convective heat transfer of supercritical water or carbon dioxide flowing in straight pipes [3-7], in some of which the effects of buoyancy were considered. So far, there have been few studies about the influences of centrifugal force on the convective heat transfer of supercritical fluid. Li et al. [8] investigated the turbulent heat transfer to near-critical water in a heated curved pipe numerically, and presented the characteristics of the heat transfer at different conditions.

\footnotetext{
* Corresponding author. Tel./fax: +86 1082543108.

E-mail address: hxl@mail.etp.ac.cn (X. Huai).
}

Hydrocarbon fuel, which works at a supercritical pressure, is very important in the active cooling system of rocket and regenerative cooling of scramjet engine as refrigerant. The hydrocarbon fuel is composed of several hundred hydrocarbons. Thus, the physical properties of the hydrocarbon fuel are more complex than those of water or carbon dioxide, and the convective heat transfer is also more complicated. Zhong et al. [9] studied the heat transfer of China No. 3 aviation kerosene at supercritical conditions experimentally. Linne et al. [2] evaluated the heat transfer of supercritical JP-7 fuel experimentally. However, they did not consider the influences of the body force on the convective heat transfer.

In this paper, a CFD software FLUENT 6.3 is used to analyze the effects of the buoyancy and centrifugal forces on the heat transfer of China No. 3 aviation kerosene at a supercritical pressure. The RNG $k-\epsilon$ two-equation turbulence model [10] that is efficient to model the flow field with secondary flow and variable physical properties is used to solve the main flow of the kerosene in a horizontal curved pipe. The Wolfstein one-equation model [11] is used to solve the low Reynolds flow at the near-wall region.

\section{Numerical methods}

\subsection{Computational models}

The China No. 3 aviation kerosene flows in a curved pipe. The geometry and the system of coordinates are shown in Fig. 1. The 


\begin{tabular}{|c|c|c|c|}
\hline \multicolumn{2}{|c|}{ Nomenclature } & $S$ & source term \\
\hline$A$ & cross-section area $\left(\mathrm{m}^{2}\right)$ & $W$ & axial velocity $(\mathrm{m} / \mathrm{s})$ \\
\hline$C_{\mathrm{p}}$ & specific heat capacity $(\mathrm{J} /(\mathrm{kg} \mathrm{K}))$ & $y$ & distance to the wall (m) \\
\hline$d$ & diameter of the pipe $(\mathrm{m})$ & & \\
\hline$f$ & fraction factors & \multicolumn{2}{|c|}{ Greek symbols } \\
\hline$h$ & heat transfer coefficients $\left(\mathrm{W} /\left(\mathrm{m}^{2} \mathrm{~K}\right)\right)$ & $\lambda$ & curvature ratios $(\lambda=r / R)$ \\
\hline$k$ & turbulent kinetic energy $\left(\mathrm{m}^{2} / \mathrm{s}^{2}\right)$ & $\mu$ & viscosity $(\mathrm{kg} /(\mathrm{m} \mathrm{s}))$ \\
\hline$K$ & thermal conductivity $(\mathrm{W} /(\mathrm{m} \mathrm{K}))$ & $v$ & kinematic viscosity $\left(\mathrm{m}^{2} / \mathrm{s}\right)$ \\
\hline $\mathrm{Nu}$ & Nusselt number $(\mathrm{Nu}=\mathrm{hd} / \mathrm{K})$ & $\rho$ & density $\left(\mathrm{kg} / \mathrm{m}^{3}\right)$ \\
\hline $\operatorname{Pr}$ & Prandtl number $\left(\operatorname{Pr}=\mu C_{\mathrm{p}} / K\right)$ & $\tau_{\mathrm{w}}$ & wall shear stress $\left(\mathrm{N} / \mathrm{m}^{2}\right)$ \\
\hline$q$ & heat flux $\left(\mathrm{W} / \mathrm{m}^{2}\right)$ & & \\
\hline$r$ & inner radius of the pipe $(\mathrm{m})$ & \multicolumn{2}{|c|}{ Subscripts } \\
\hline$R$ & curvature radius of the pipe $(\mathrm{m})$ & $\mathrm{b}$ & bulk quantity \\
\hline $\operatorname{Re}$ & Reynolds number $(\operatorname{Re}=W d / v)$ & c & curved pipe \\
\hline $\mathrm{Re}_{\mathrm{y}}$ & $\begin{array}{l}\text { wall-distance-based turbulent Reynolds number } \\
\left(\operatorname{Re}_{\mathrm{y}}=\rho y \sqrt{k} / \mu\right)\end{array}$ & w & wall \\
\hline
\end{tabular}

radius of the pipe is $r$ with a curvature radius of $R$. The kerosene flows along the $z$ direction at a supercritical pressure. The flow and heat transfer of the kerosene flowing in this curved pipe can be affected by the buoyancy and centrifugal force.

The buoyancy is considered in the momentum equation by a constant value of $10 \mathrm{~m} / \mathrm{s}^{2}$ at negative $y$-coordinate. The centrifugal force is introduced by defining the source term $S_{\mathrm{i}}$ of the momentum equation. It is given:

$S=\rho \frac{W^{2}}{R+d R}$

where $W$ is the axial velocity, $R$ is the curvature radius of the pipe, $d R$ is the coordinates values ( $x$-coordinate) in the direction of centrifugal force in the Cartesian coordinates.

In the fully turbulent region ( $\left.\operatorname{Re}_{\mathrm{y}}>200\right)$, the RNG $k-\epsilon$ model is employed. In the viscosity-affected near-wall region $\left(\operatorname{Re}_{\mathrm{y}} \leq 200\right)$, enhanced wall treatment (one-equation model of Wolfstein) is used to fit the low Reynolds flow [12].

\subsection{Boundary conditions}

In fact, the China No. 3 aviation kerosene flows in a horizontal coiled pipe. And the pitch of the coil pipe is omitted. So, the coiled pipe can be taken as a curved pipe to be investigated. The boundary conditions are shown as follows:

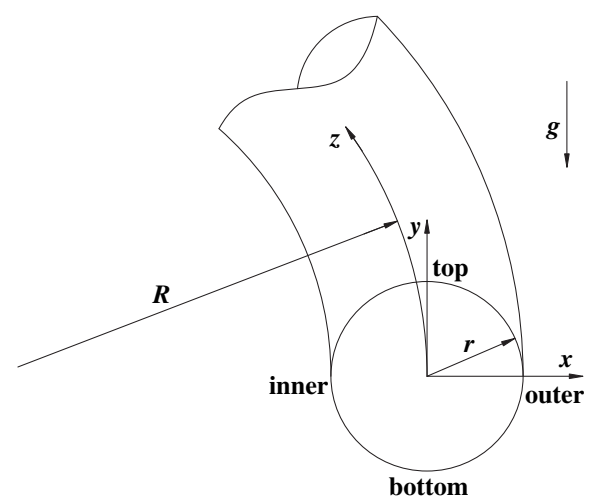

Fig. 1. Curved pipe coordinates system.
(1) The inner diameter of the pipe is $12 \mathrm{~mm}$, the length of unheated section from the inlet is $0.5 \mathrm{~m}$, and the length of heated section is $4.5 \mathrm{~m}$.

(2) The inlet pressure is $4 \mathrm{MPa}$, and the inlet temperature is $300 \mathrm{~K}$. An outflow boundary condition is applied to the outlet.

(3) The mass flow rate is $0.1 \mathrm{~kg} / \mathrm{s}$, and the inlet Reynolds number is 8700.

(4) The distribution of the wall heat flux $q_{\mathrm{w}}(x)$ along the axial direction is shown in Fig. 2, which refers to the true distribution of heat flux of scramjet [13].

\subsection{Data analysis}

The average heat transfer coefficients are defined as follows:

$h=\frac{q}{\overline{T_{\mathrm{W}}}-T_{\mathrm{b}}}$

where $q$ is the wall heat flux along the axial direction (shown in Fig. 2), $\overline{T_{\mathrm{w}}}$ is the average temperature of the wall on the circumference, $T_{\mathrm{b}}$ is the cross-sectional bulk temperature of the fluid calculated by

$T_{\mathrm{b}}=\left(\int_{A} T_{\mathrm{i}} \mathrm{d} A\right) / A$

The friction factors of the channel are given by

$f=\frac{\overline{\tau_{\mathrm{w}}}}{0.5 \bar{\rho} \bar{W}^{2}}$

where $\overline{\tau_{w}}$ is the average wall shear stress around the channel circumference, $\bar{\rho}$ is the average density according to the bulk temperature $T_{\mathrm{b}}, \bar{W}$ is the average axial velocity in a cross section.

\subsection{Analysis of the grid independence}

The grid dependence study is conducted to confirm the grid independence. The grids of the cross section are shown in Fig. 3. The calculated results are compared among different grids distribution (cross-sectional $\times$ axial) of $2145 \times 500,2145 \times 800$, $2145 \times 1000,1680 \times 800$, and $2628 \times 800$ with a curvature radius $R$ of $0.3 \mathrm{~m}$. By the analysis of the heat transfer coefficients and temperature at different grids, the maximum deviation of the grids 


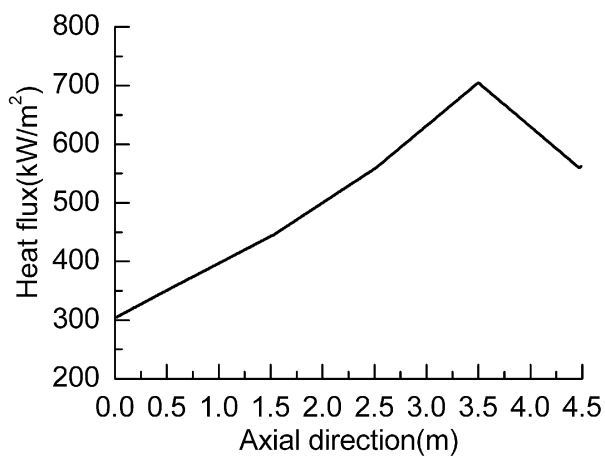

Fig. 2. Distributions of the wall heat flux along the axial direction.

$2145 \times 500$ with the other grids is $6 \%$, and the maximum deviation among said other grids is within $1 \%$. Thus, the girds of $2145 \times 800$ are selected as the baseline for the rest of studies.

\section{Thermophysical properties of the kerosene at supercritical pressure}

The thermophysical properties of China No. 3 aviation kerosene are calculated by the NIST Supertrapp software [14] with a 10species surrogate [9]. The computed critical temperature and pressure are $660 \mathrm{~K}$ and $2.4 \mathrm{MPa}$, respectively. While the measured critical temperature and pressure are $646 \mathrm{~K}$ and $2.4 \mathrm{MPa}$ [15], respectively. Fig. 4 shows the variation of the computed thermophysical properties of the kerosene with temperature at the supercritical pressure of $4 \mathrm{MPa}$. The pseudo-critical temperature is $720 \mathrm{~K}$ at this pressure. The thermophysical properties of the kerosene are different from those of water or carbon dioxide at supercritical pressure because the kerosene is composed of lots of organic compounds.

Comparing the thermophysical properties of the kerosene with those of the water or carbon dioxide at supercritical pressure, there are some differences listed as follows: (1) The specific heat capacity of the kerosene continuously rises before the pseudo-critical temperature, and still be high after the pseudo-critical point. However, the specific heat capacity of the water or carbon dioxide only has a maximum value at the pseudo-critical point, and the

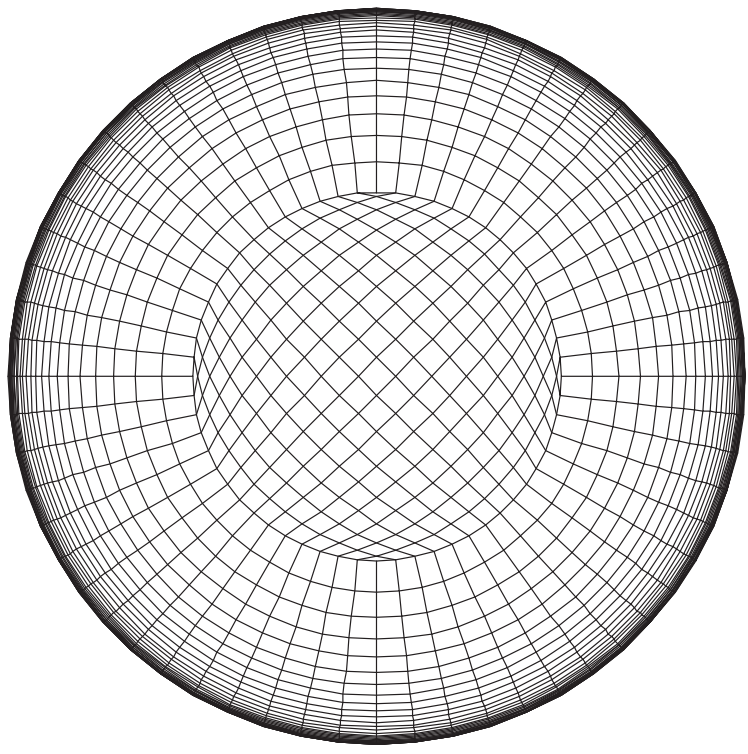

Fig. 3. Meshes of the cross section.

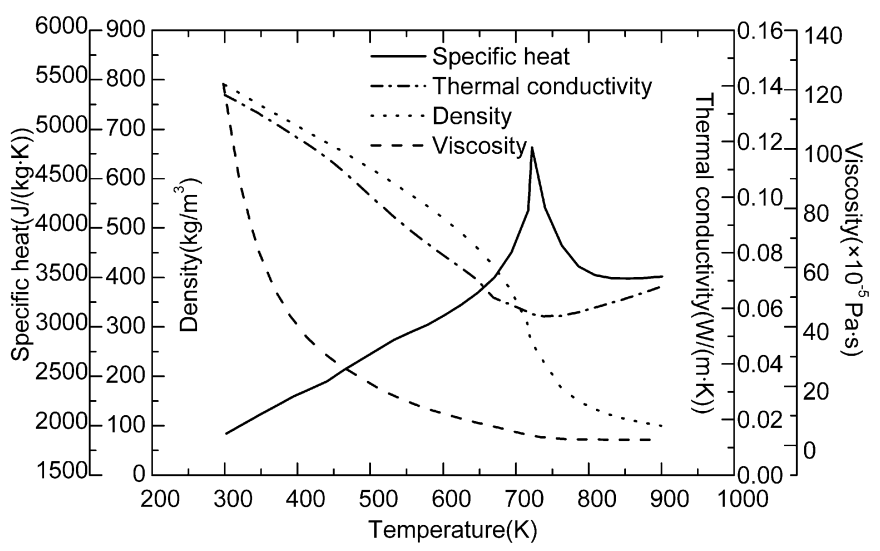

Fig. 4. Thermophysical properties of the kerosene at a supercritical pressure of $4 \mathrm{MPa}$.

values have little change before or after that point. (2) The thermal conductivity of the kerosene almost decreases linearly with the increase of the temperature, and there is no acute change at the pseudo-critical point. (3) The viscosity of the kerosene decreases rapidly before the pseudo-critical temperature, but changes flatly near the pseudo-critical point. The differences of the kerosene thermophysical properties mentioned above cause more complicated convective heat transfer.

\section{Result discussion}

\subsection{Analysis of the computation}

The accuracy and reliability of present computation technique should be checked before this study. There were no experimental data about the dual influences of the buoyancy and centrifugal force on the convective heat transfer of the supercritical fluid, even the influences of only centrifugal force were not found. Thus, the computational results with current methods are compared with previous experimental data of common water flowing in curved pipes to confirm the precision about the effects of the centrifugal force, and are compared with previous experimental data of supercritical water flowing in a horizontal straight pipe to confirm the precision about the influences of the buoyancy, respectively.

The average Nusselt number under the influences of the centrifugal force are compared between the present computation and the previous investigations by Rogers and Mayhew [16], Mori and Nakayama [17] and Shchukin [18]. The results of the comparisons are shown in Fig. 5. The maximum deviation between the present prediction and the previous experimental studies is less than $5 \%$.

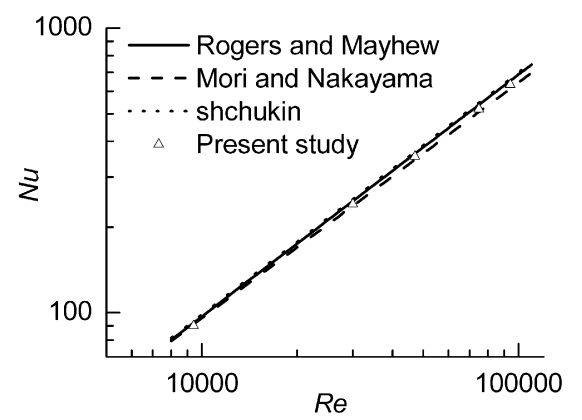

Fig. 5. Comparisons of the computed Nusselt number with the empirical formulas $(\operatorname{Pr}=7, \lambda=0.075)$ 
The empirical formula presented by Rogers and Mayhew's experimental work is

$\mathrm{Nu}_{\mathrm{c}}=0.023 \operatorname{Re}^{0.85} \operatorname{Pr}^{0.4} \lambda^{0.1}$

where $\mathrm{Nu}_{\mathrm{c}}$ is the average Nusselt number for a curved pipe, $\lambda(=r / R)$ is the curvature ratios of the curved pipe.

Mori and Nakayama's experimental data are presented by

$\mathrm{Nu}_{\mathrm{c}}=\frac{1}{41} \operatorname{Re}^{\frac{5}{6}} \lambda^{\frac{1}{12}}\left[1+\frac{0.061}{\left(\operatorname{Re} \lambda^{2.5}\right)^{\frac{1}{6}}}\right]$

The empirical formula presented by Shchukin is

$\mathrm{Nu}_{\mathrm{c}}=0.0266\left(\operatorname{Re}^{0.85} \lambda^{0.15}+0.225 \lambda^{1.55}\right) \operatorname{Pr}^{0.4}$

The heat transfer coefficients and the wall temperature of the supercritical water flowing in a horizontal straight pipe are compared between the present study and the previous experimental data presented by Yamagata [3]. The results of the comparisons are shown in Fig. 6. The current calculation is in good agreement with the previous experimental data. The maximum deviation of the wall temperature between the present prediction and the previous experimental studies is less than $2 \%$. However, no definite conclusions can be drawn with respect to the accuracy of the present heat transfer coefficients, as the uncertainty in the experimental data near the pseudo-critical temperature is large. The present computation shows that the buoyancy causes a strong secondary flow, and that the heat transfer coefficients on the bottom wall are higher than those on the top wall, especially at the pseudo-critical point.

\subsection{Effect of the body force}

First, the flow and convective heat transfer without body force are studied to reflect the supercritical characteristics. The distributions of the heat transfer coefficients and the wall temperature without body force along the axial direction are shown in Fig. 7. The Reynolds number and Prandtl number are shown in Fig. 8. The heat transfer coefficients increase as the rise of Reynolds number at the initial heated section $(0<L<2.5 \mathrm{~m})$. However, the heat transfer coefficients turn down when the wall temperature is higher than the pseudo-critical temperature of $720 \mathrm{~K}$, though the Reynolds number rises continuously. This indicates the deterioration of the heat transfer. Then, the heat transfer coefficients increase again after the location of $L=3 \mathrm{~m}$, and rise rapidly along the flow

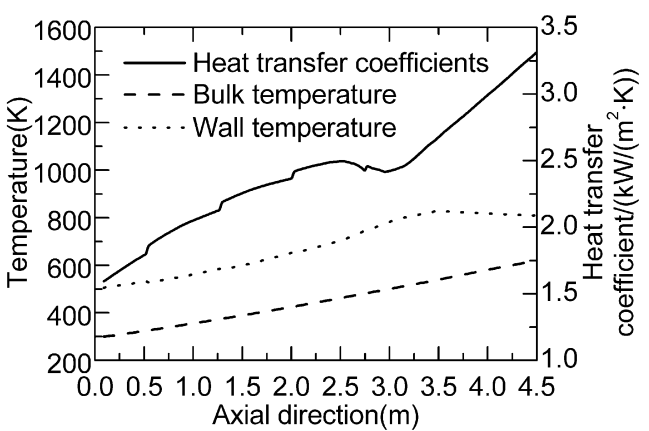

Fig. 7. Distributions of the wall and bulk temperature and heat transfer coefficients without body force.

direction, which corresponds to the enhancement of the heat transfer. These phenomena are also observed in the study of Zhong [9]. Fig. 8 shows that the Reynolds number increases by at least one order of magnitude and that the Prandtl number decreases by one order of magnitude. The results show the complicated characteristics of the convective heat transfer of the kerosene.

Then the calculation with body force is carried out. The average heat transfer coefficients at different conditions are compared in Fig. 9. The heat transfer coefficients with only buoyancy are slightly higher than those without body force after the wall temperature is over the pseudo-critical temperature at the axial location of $2.75 \mathrm{~m}$. However, the heat transfer coefficients with only centrifugal force are enhanced from the entry of the pipe. The heat transfer coefficients with buoyancy and centrifugal force are the same as those with only centrifugal force, and the lines of them are coincidence in the figure. Thus, the buoyancy can be omitted when the centrifugal force exists in this study. The enhancement rates of the heat transfer coefficients with only centrifugal force rise along the axial direction. After the wall temperature reaches the pseudo-critical temperature, the enhancement rates are over $12 \%$, and have little change. The starting points of the heat transfer enhancement delay along the axial direction under the effects of the centrifugal force.

Fig. 10 shows the friction factors affected by the body force. The friction factors reduce along the axial direction. Then, they are flat after the location of $3.5 \mathrm{~m}$ because the wall temperature becomes flat. The friction factors with only buoyancy and those without body force have little differences before the location of $2.75 \mathrm{~m}$, which location is corresponding to the pseudo-critical temperature on the wall. After that, the friction factors with only buoyancy are higher
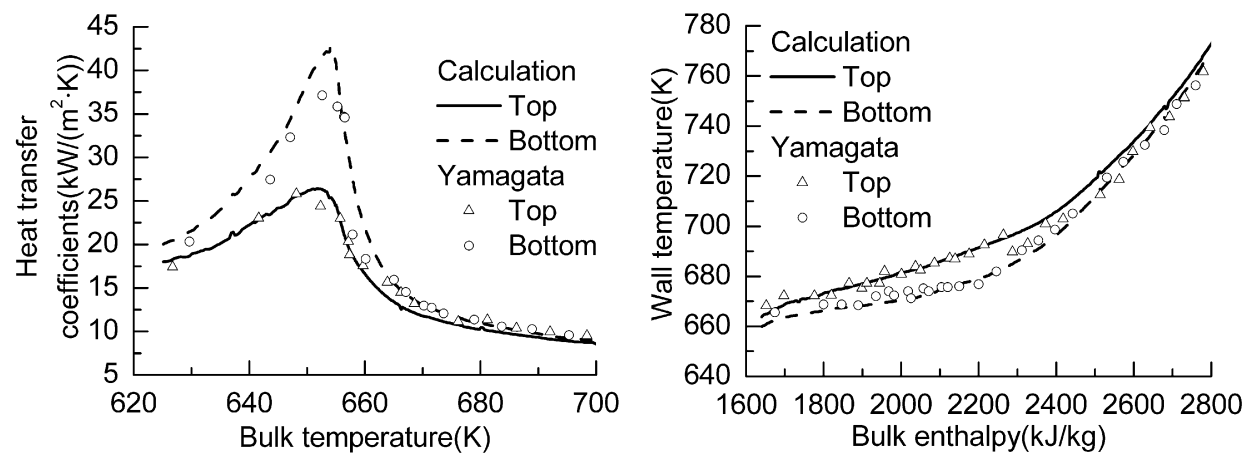

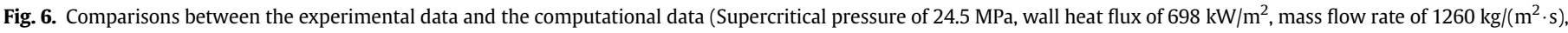
inner diameter of $7.5 \mathrm{~mm}$ ). 


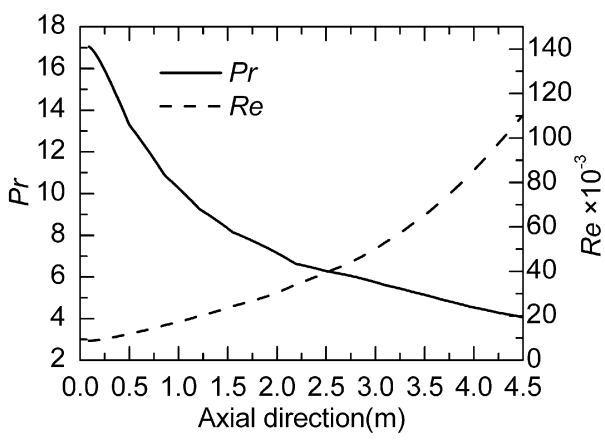

Fig. 8. Distributions of $\operatorname{Pr}$ and Re without body force.

than those without body force. The friction factors with only centrifugal force and those with buoyancy and centrifugal force are the same throughout the process, which are coincidence in the figure. The friction factors with only centrifugal force are higher than those without body force. And the difference values between them hardly change along the flow direction, and the friction factors with only centrifugal force are over $14 \%$ higher than those without body force. The centrifugal force causes a strong secondary flow perpendicular to the main flow direction, enhances the disturbance. Consequently, the heat transfer is increased. However, it is a pity that the friction factors are also enhanced.

The circumferential heat transfer coefficients at different sections are shown in Fig. 11. Fig. 11(a) shows the heat transfer coefficients with only buoyancy. The buoyancy causes a secondary flow along the gravity direction, which enhances the heat transfer on the bottom and decreases the heat transfer on the top. The heat transfer coefficients on the top at the location of $3 \mathrm{~m}$ are below those at the location of $2 \mathrm{~m}$. Thus, the deterioration of heat transfer is magnified on the top under the effects of the buoyancy. Fig. 11(b) shows the heat transfer coefficients with only centrifugal force. The centrifugal force with a curvature ratio of 0.02 also induces a secondary flow along the centrifugal force direction. Obviously, the centrifugal force makes the average heat transfer coefficients enhanced. The heat transfer coefficients at the centrifugal force direction and its two sides become strong, and only those at the opposite direction of the centrifugal force are weak. The dual effects of the buoyancy and centrifugal force on the convective heat transfer are shown in Fig. 11(c). The dual effects of the buoyancy and centrifugal force are complex. The effects of the centrifugal force are predominant. Although the average heat transfer coefficients with the dual effects are the same as those with only centrifugal force, the lowest points of the heat transfer coefficients are different. The lowest points of the heat transfer coefficients with dual effects gradually offset to the

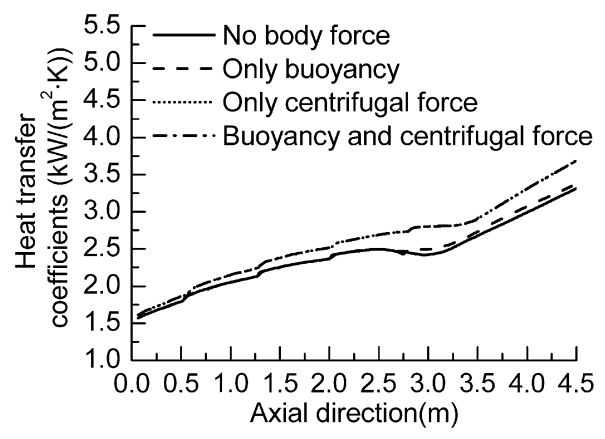

Fig. 9. Comparisons of the heat transfer coefficients.

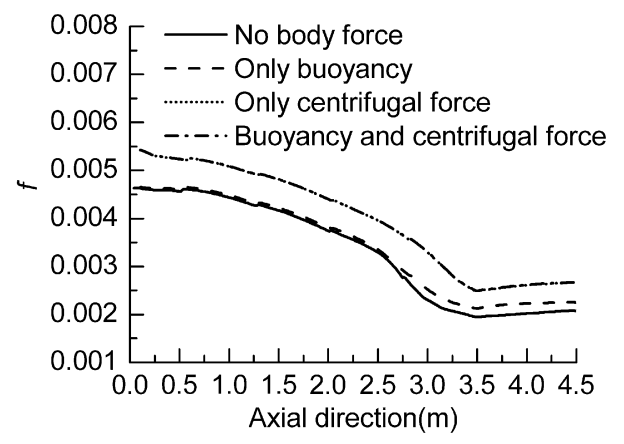

Fig. 10. Comparisons of the friction factors.

top along the flow direction. This indicates that the effects of the buoyancy become strong related to those of the centrifugal force, because that the density differences become large along the flow direction.

\subsection{Effect of the curvature}

Calculation is also carried out to study the effects of the curvature ratios on the flow and heat transfer of the supercritical
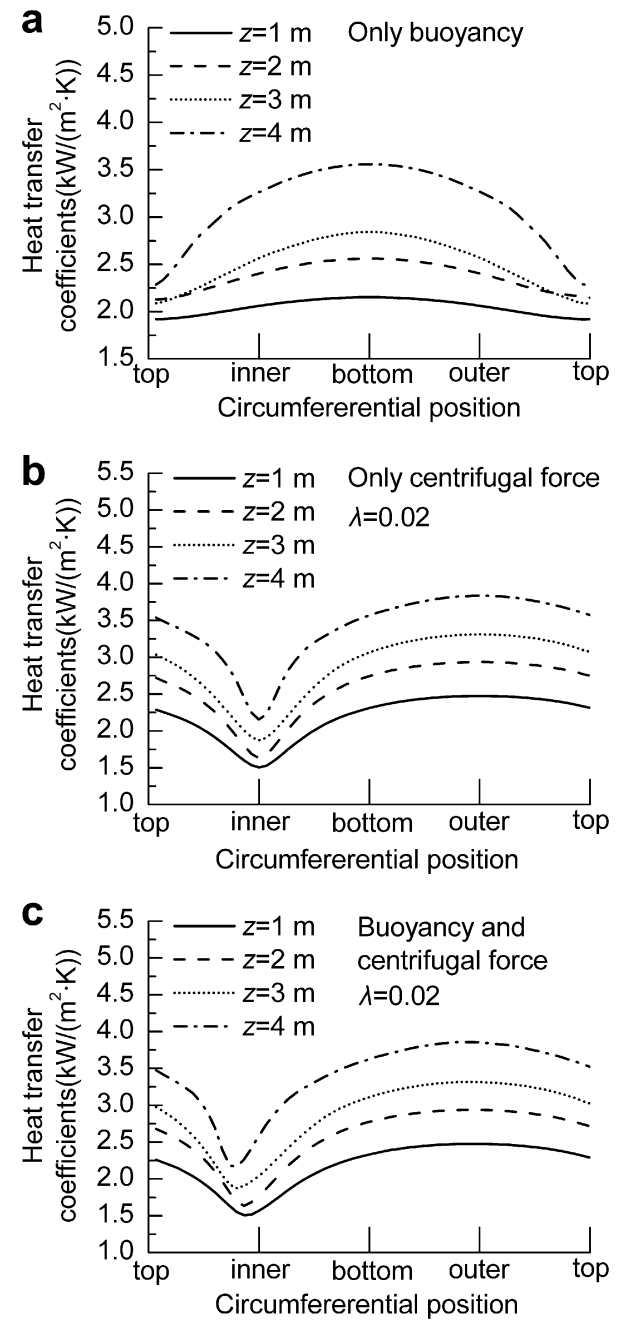

Fig. 11. Distributions of the circumferential heat transfer coefficients at different sections. 


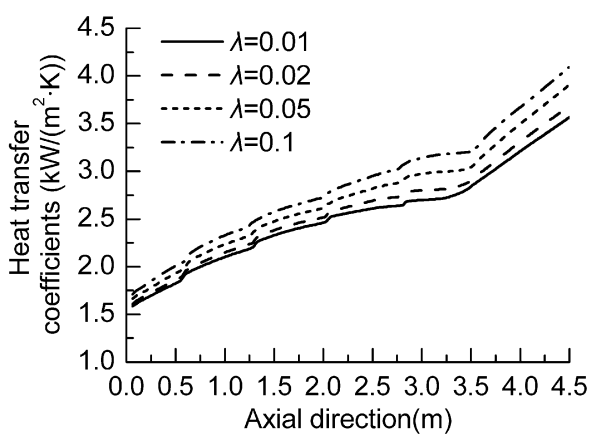

Fig. 12. Comparisons of the heat transfer coefficients with different curvature ratios.

kerosene. The curvature ratios are $0.01,0.02,0.05$ and 0.1 , respectively. The effects of curvature ratios on the average heat transfer coefficients and the friction factors are shown in Figs. 12 and 13. The average heat transfer coefficients increase as the curvature ratios increasing. However, the start points of the heat transfer enhancement hardly change. There is not deterioration of the heat transfer at the effects of the centrifugal force. The friction factors also increase as the curvature ratios increasing, which are caused by the strong secondary flow at the sections. The improvement of the friction factors is larger with the increase of the curvature ratios in the inlet of the pipe, and then it become small gradually. Furthermore, the turning points of the friction factors only occur at the peak of the wall heat flux. The friction factors become flat after that point.

The circumferential heat transfer coefficients are given to investigate the local heat transfer with different curvature ratios. The local circumferential heat transfer coefficients with different curvature ratios are shown in Fig. 14. The lowest points of the heat transfer occur near the inner side. The peak points of the heat transfer occur near the outer side. However, the heat transfer coefficients are high at most of the areas. Furthermore,

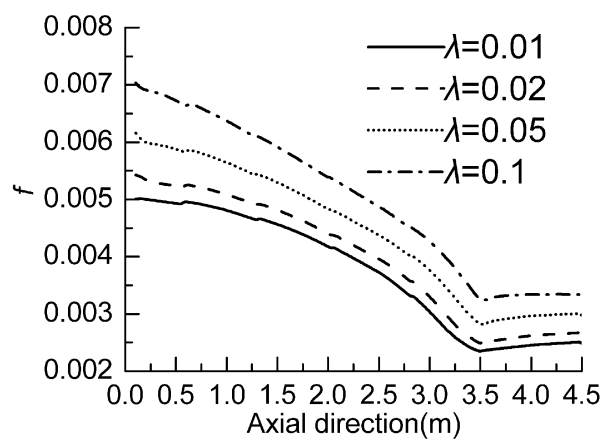

Fig. 13. Comparisons of the friction factors with different curvature ratios.

the lowest points of the heat transfer are gradually close to the top along the flow direction when the curvature ratios are less than 0.05 . Those indicate that the effects of the buoyancy become strong along the flow direction at the supercritical pressure. When the curvature ratios are greater than 0.05 , the lowest and the highest points of the heat transfer coefficients hardly change along the flow direction. That is to say, the effects of buoyancy do not enhance relative to those of centrifugal force along the flow direction.

The secondary flow caused by the centrifugal force is not only related to the velocity differences, but also related to the density differences. The centrifugal force is proportional to the square of the velocity, but the rapid increase of the velocity caused by the variation of the density at the supercritical pressure do not enhance the effects of the centrifugal on the secondary flow direction when the curvature ratios are less than 0.05 . On the contrary, the effects of the buoyancy become large along the flow direction because of the large changes of the density. While the curvature ratios are more than 0.05 , the effects of the centrifugal force begin to become large along the flow direction.
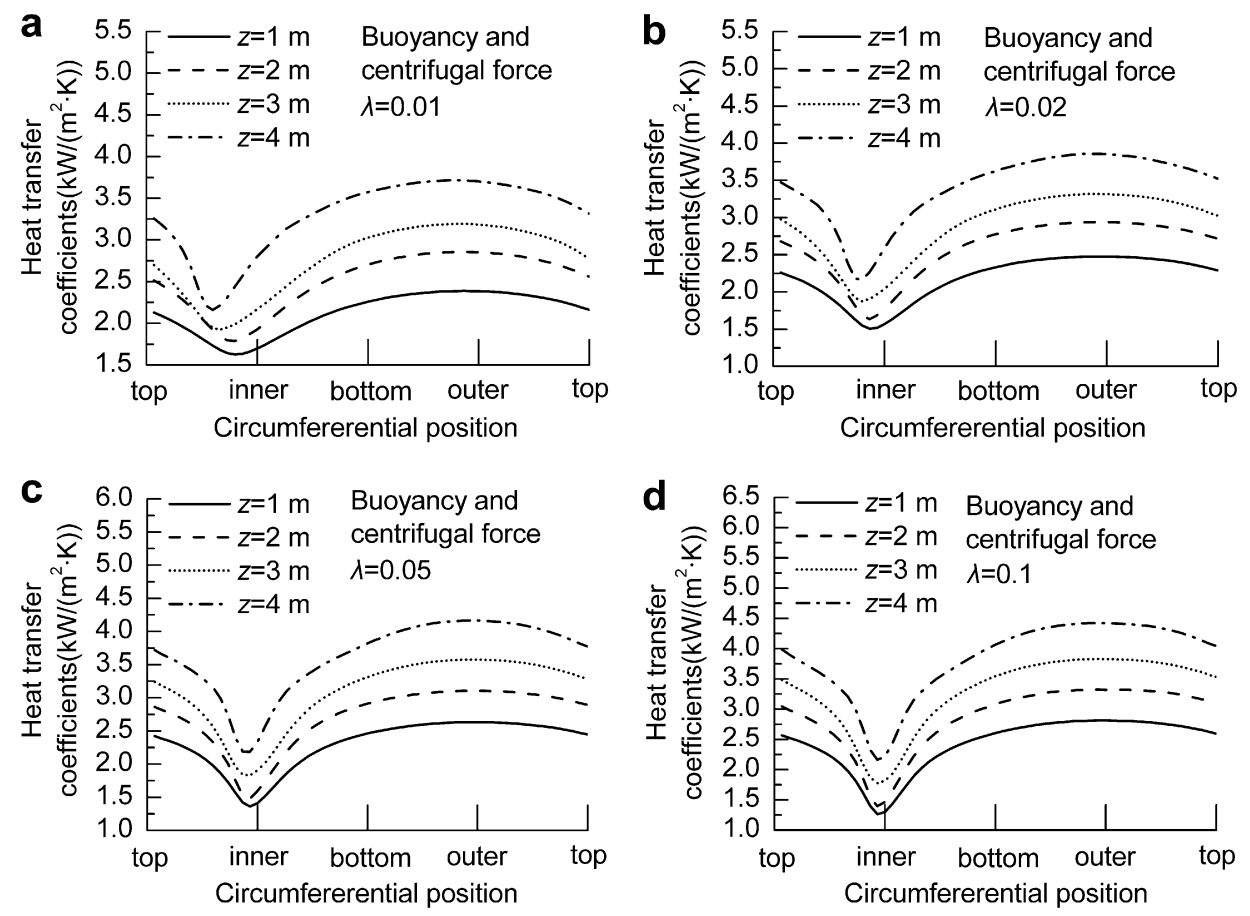

Fig. 14. Distributions of the circumferential heat transfer coefficients with different curvature ratios. 


\section{Conclusions}

Flow and heat transfer of the China No.3 aviation kerosene in a curved pipe at a supercritical pressure of $4 \mathrm{MPa}$ are numerically investigated by the FVM. The thermophysical properties of the supercritical kerosene are described and compared with those of the supercritical water or dioxide carbon. The dual effects of the buoyancy and the centrifugal force are considered in this study.

Because of the large variation of the thermophysical properties of the supercritical kerosene, the heat transfer is enhanced after the wall temperature is over the pseudo-critical temperature. The buoyancy has little effects on the heat transfer and flow before the wall temperature is lower than the pseudo-critical temperature. After that point, the large density difference has complicated influences on the heat transfer and flow. However, the centrifugal force causes a strong secondary flow, which not only enhances the heat transfer largely, but also increases the friction factors greatly throughout the flow process. Furthermore, the centrifugal force is dominant in the mixed heat transfer under this calculational condition. The turning points of the friction factors are corresponding to the peak of wall heat flux. The curvature ratios have more effects on the heat transfer and flow. The bigger the curvature ratios are, the stronger the heat transfer coefficients and friction factors are.

The secondary flow caused by the body force leads to a large variation of the circumferential heat transfer. The heat transfer enhances in the secondary flow direction, and decreases at the opposite position. Moreover, the secondary flow induced by the centrifugal force makes the heat transfer enhanced at most of the circumference.

Although the velocity occupies an important position in the centrifugal force, the rise of the velocity caused by the variation of the density does not improve the scale of the centrifugal force during the heat transfer with the dual effects when the curvature ratios are less than 0.05 . In contrary, the effects of the buoyancy on the heat transfer are enhanced along the flow direction because of the extensive variation of the density.

\section{Acknowledgements}

The authors gratefully acknowledge the financial support of the Chinese National key Foundation Research Subject (No.
G2010CB227302) and the National Natural Science Foundation of China (No.50776087 and No.10672169)

\section{References}

[1] J. Yang, Y. Oka, Y. Ishiwatari, J. Liu, J. Yoo, Numerical investigation of heat transfer in upward flows of supercritical water in circular tubes and tight fuel rod bundles. Nuclear Engineering and Design 237 (4) (2007) 420-430.

[2] D.L. Linne, M.L. Meyer, T. Edwards, D.A. Eitman, Evaluation of Heat Transfer and Thermal Stability of Supercritical JP-7 Fuel, NASA Technical Memorandum 107485, AIAA-97-3041.

[3] K. Yamagata, K. Nishikawa, S. Hasegawa, T. Fujii, S. Yoshida, Forced convective heat transfer to supercritical water flowing in tubes. International Journal of Heat and Mass Transfer 15 (12) (1972) 2575-2593.

[4] M. Bazargan, D. Fraser, V. Chatoorgan, Effect of buoyancy on heat transfer in supercritical water flow in a horizontal round tube. Journal of Heat Transfer 127 (2005) 897-902.

[5] F. Roelofs, CFD Analyses of Heat Transfer to Supercritical Water Flowing Vertically Upward in a Tube, NRG, 21353/04.60811/P.

[6] X.R. Zhang, H. Yamaguchi, Forced convection heat transfer of supercritical $\mathrm{CO}_{2}$ in a horizontal circular tube. Journal of Supercritical Fluids 41 (3) (2007) $412-420$.

[7] S.M. Liao, T.S. Zhao, Measurements of heat transfer coefficients from supercritical carbon dioxide flowing in horizontal mini/micro channels. ASME Journal of Heat Transfer 124 (2002) 413-420.

[8] L.J. Li, X. Lin, M.A. Ebadian, Turbulent heat transfer to near-critical water in a heated curved pipe under the conditions of mixed convection. International Journal of Heat and Mass Transfer 42 (16) (1999) 3147-3158.

[9] F. Zhong, X. Fan, G. Yu, J. Li, X. Lu, Heat Transfer of Aviation Kerosene at Supercritical Conditions, AIAA-2008-4615.

[10] V. Yakhot, S.A. Orszag, Renormalization group analysis of turbulence: I. basic theory. Journal of Scientific Computing 1 (1) (1986) 1-51.

[11] M. Wolfstein, The velocity and temperature distribution of one-dimensional flow with turbulence augmentation and pressure gradient. International Journal of Heat and Mass Transfer 12 (2) (1969) 301-318.

[12] Ansys Fluent, FLUENT 6.3 User's Guide. Fluent Inc, USA, 2006, p. 10.

[13] L. Zheng, B. Sun, 2D thermal environment numerical simulation of scramjets. Journal of Aerospace Power 22 (5) (2007) 823-828.

[14] J.F. Ely, M.L. Huber, NIST Standard Reference Database 4-NIST Thermophysical Properties of Hydrocarbon Mixtures. National Institute of Standards, Gaithersburg, MD, Feb, 1990.

[15] Q. Sun, Z. Mi, X. Zhang, Determination of critical properties $\left(t_{\mathrm{c}}, p_{\mathrm{c}}\right)$ of endothermic hydrocarbon fuels-PR-3 and simulated JP-7. Journal of Fuel Chemistry and Technology 34 (4) (2006) 466-470.

[16] G.F.C. Rogers, Y.R. Mayhew, Heat transfer and pressure loss in helically coiled tubes with turbulent flow. International Journal of Heat and Mass Transfer 7 (1964) 1207-1216.

[17] Y. Mori, W. Nakayama, Study on forced convective heat transfer in curved pipes (2nd report, turbulent). International Journal of Heat and Mass Transfer 10 (1967) 37-59.

[18] V.K. Schhukin, Correlation of experimental data on heat transfer in curved pipes. Thermal Engineering 16 (1969) 72-76. 